SLAC-PUB-10088

BABAR-PROC-03/013

hep-ex/0000

August, 2003

\title{
Rare Radiative and Leptonic $B$ Decays: Recent Results from BABAR and Belle
}

\author{
A.M. Eisner \\ University of California at Santa Cruz, Institute for Particle Physics \\ 1156 High Street, Santa Cruz, CA 95064, USA
}

\begin{abstract}
Recent $B A B A R$ and Belle results on rare $B$ decays involving photons or lepton pairs are reviewed. New preliminary limits from both experiments for $B \rightarrow \rho \gamma$ and $B^{0} \rightarrow \omega \gamma$, and from BABAR for $B^{-} \rightarrow K^{-} \nu \bar{\nu}$ and $B^{+} \rightarrow \tau^{+} \nu_{\tau}$ are presented. Also summarized from the past year are Belle measurements of $B \rightarrow X_{s} \ell^{+} \ell^{-}$and $B^{0} \rightarrow K_{2}^{*}(1430)^{0} \gamma$, preliminary BABAR measurements of $B \rightarrow$ $X_{s} \gamma$, and preliminary results from $B A B A R$ and Belle on $B \rightarrow K^{(*)} \ell^{+} \ell^{-}$. No significant deviations from Standard Model predictions have yet been found.
\end{abstract}

Contributed to the Proceedings of the XXXVIII Rencontres de Moriond, Electroweak Interactions and Unified Theories, 3/15/2003-3/22/2003, Les Arcs, France

Stanford Linear Accelerator Center, Stanford University, Stanford, CA 94309

Work supported in part by Department of Energy contracts DE-AC03-76SF00515 and DE-FG03-92ER40689. 


\section{Introduction}

This review covers results from BABAR and Belle since the 2002 Moriond-EW conference in the area of rare $B$ decays involving photons or lepton pairs. In particular, the final states to be presented are $\rho \gamma$ and $\omega \gamma$ (new results from both experiments), $K^{*}(1430) \gamma$ (Belle, published), $X_{s} \gamma(B A B A R$, of ICHEP-2002 vintage), $K^{(*)} \ell^{+} \ell^{-}$(both experiments, ICHEP-2002), $X_{s} \ell^{+} \ell^{-}$(Belle, published), $K^{-} \nu \bar{\nu}$ (new result from $B A B A R$ ) and $\tau^{+} \nu_{\tau}$ (new results from $B A B A R$ ). Here $X_{s}$ refers to inclusvie strange hadronic states, and $\ell^{+} \ell^{-}$to either a di-muon or di-electron pair.

None of these decays occurs at tree level; rather, all involve internal loops or boxes or (for the purely leptonic $\tau^{+} \nu_{\tau}$ decay) $u \bar{b}$ annihilation. In all cases the predicted branching fractions are low, and in most the theoretical uncertainties in the Standard Model ("SM") are low. (Because of fragmentation effects, uncertainties are larger whenever a specific hadron appears in the final state.) Thus these are good places to look for non-standard contributions, with the potential for new particles showing up virtually in the loops. For example, Figure 1 illustrates one of the two "radiative penguin" diagrams responsible for $B \rightarrow X_{s} \gamma$ in the Standard Model, while Figure 2 shows penguin and box diagrams for $B \rightarrow X_{s} \ell^{+} \ell^{-}$. Of course, there are QCD corrections to these lowest-order amplitudes.

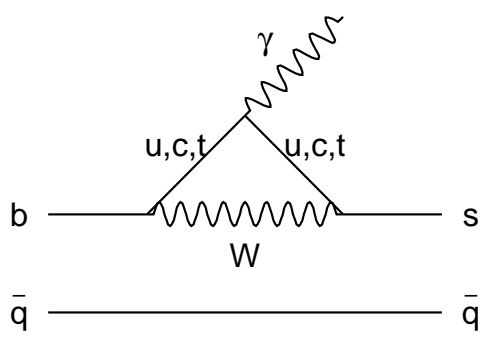

Figure 1: $B \rightarrow X_{s} \gamma$ in the Standard Model (penguin diagram)

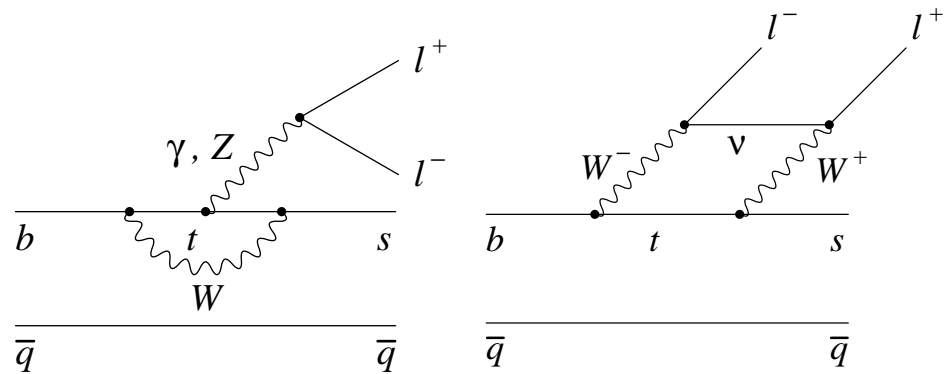

Figure 2: $B \rightarrow X_{s} \ell^{+} \ell^{-}$in the Standard Model (penguin and box diagrams)

\section{Radiative B Decays}

Next-to-leading order (NLO) predictions for $B \rightarrow X_{s} \gamma$ in the Standard Model have been made to about $10 \%$ precision 1, 2]. The easiest component of this to measure is the exclusive decay $B \rightarrow K^{*}(892) \gamma$, which has a branching fraction of $\sim 4 \times 10^{-5}$, and has been determined in each charge state to about $10 \%$ precision [3]. Unfortunately, predictions are much less certain, since hadronization is involved.

This review presents results which extend the experimental knowledge in several directions: to higher $K^{*}$ resonances, to the inclusive $B \rightarrow X_{s} \gamma$ process, and to searches for $B \rightarrow X_{d} \gamma$. The latter proceeds in the Standard Model via a diagram analogous to Figure 1 with the $s$ quark replaced by a $d$. Because the amplitude is dominated by a $t$ quark in the loop, the $B \rightarrow X_{d} \gamma$ yield is reduced by $\sim\left(\left|V_{t d}\right| /\left|V_{t s}\right|\right)^{2} \sim 0.04$ compared to $B \rightarrow X_{s} \gamma$. As with $B \rightarrow X_{s} \gamma$, the first searches are for exclusive states, the $\rho$ and the $\omega$. 


\subsection{Searches for $B^{+} \rightarrow \rho^{+} \gamma, B^{0} \rightarrow \rho^{0} \gamma$ and $B^{0} \rightarrow \omega \gamma$}

Predictions for $\mathcal{B}\left(B^{+} \rightarrow \rho^{+} \gamma\right)$ in the Standard Model [4, 5] are $\sim 0.9$ to $1.5 \times 10^{-6}$. Using isospin symmetry and the quark model, one expects $\mathcal{B}\left(B^{0} \rightarrow \rho^{0} \gamma\right)$ and $\mathcal{B}\left(B^{0} \rightarrow \omega \gamma\right)$ each $\approx \mathcal{B}\left(B^{+} \rightarrow\right.$ $\left.\rho^{+} \gamma\right) / 2$. BABAR [6] and Belle [7] are reporting updated searches for these three decays.

Backgrounds for these measurements are:

- Continuum $(u \bar{u}, d \bar{d}, s \bar{s}, c \bar{c})$ events with the high-energy photon mostly from $\pi^{0}(\eta)$ decay or initial state radiation ("ISR"). These events have a jetlike topology.

- For $B \rightarrow \rho \gamma: B \rightarrow K^{*} \gamma$ with a $K$ misidentified as a $\pi$. This needs to be suppressed, because $\mathcal{B}\left(B \rightarrow K^{*} \gamma\right)$ is at least 25 times larger than the $\mathcal{B}$ expected for signal.

- Mainly for $B^{+} \rightarrow \rho^{+} \gamma$ : a small contribution from $B \rightarrow \rho(\omega) \pi^{0}$.

BABAR (Belle) combines topological quantities into a neural net (Fisher discriminant) to reduce continuum background. Particle-identification (PID) and kinematic criteria are used to suppress $B \rightarrow K^{*} \gamma$. In particular, BABAR achieves very good rejection using the DIRC [8] plus $\mathrm{dE} / \mathrm{dx}$; as illustrated in their paper [6], the BABAR $K$-to- $\pi$ misidentification probability in nearly all of the relevant region is determined from control samples to be less than $1 \%$.

Results for exclusive $B$ decays are typically presented using the following kinematic variables. If $\left(E_{B}^{*}, \vec{p}_{B}^{*}\right)$ is the four-momentum of a reconstructed $B$ candidate in the overall CM $(\Upsilon(4 S))$ frame, we define

$$
\begin{gathered}
\Delta E^{*} \equiv E_{B}^{*}-E_{\text {beam }}^{*}, \\
m_{E S}\left(\text { or } M_{b c}\right) \equiv \sqrt{E_{\text {beam }}^{*}-p_{B}^{* 2}} .
\end{gathered}
$$

The latter is called the energy-substituted (BABAR) or beam-constrained (Belle) mass. Signal events peak at $\Delta E^{*}$ near 0 and $m_{E S}$ near $M_{B}$ (above $\left.\approx 5.27 \mathrm{GeV} / c^{2}\right)$; whereas continuum background lacks peaks, and is usually fit in $m_{E S}$ by a single-parameter "ARGUS" threshold function 9 .

Signal extraction in $B A B A R$ uses an unbinned maximum likelihood fit in $m_{E S}, \Delta E^{*}$ and (for $\rho \gamma) m_{\pi \pi}$. As is standard in most such BABAR analysis, a signal region is "blinded" (not looked at) until all cuts and procedures are finalized. Figure 3 shows the BABAR data.

Neither BABAR [6] nor Belle [7] sees any evidence for a signal. Table 1]shows upper limits, along with those from an earlier search. The Belle and BABAR limits allow for systematic uncertainties, using a method based on Cousins and Highland [10].

Table 1: Upper limits for $B \rightarrow \rho \gamma$ and $B^{0} \rightarrow \omega \gamma$.

\begin{tabular}{|l|c|c|c|c|}
\hline & \multirow{2}{*}{$\begin{array}{c}\int \mathcal{L} d t \\
\text { Experiment }\end{array}$} & \multicolumn{3}{|c|}{$90 \%$ CL Upper Limits on $\mathcal{B}$} \\
\cline { 3 - 5 } & $\left(\mathrm{fb}^{-1}\right)$ & $B^{0} \rightarrow \rho^{0} \gamma$ & $B^{+} \rightarrow \rho^{+} \gamma$ & $B^{0} \rightarrow \omega \gamma$ \\
\hline CLEO-II [1] & 9.2 & $17 \times 10^{-6}$ & $13 \times 10^{-6}$ & $9.2 \times 10^{-6}$ \\
Belle [7] & 78 & $2.6 \times 10^{-6}$ & $2.7 \times 10^{-6}$ & $4.4 \times 10^{-6}$ \\
BABAR [6] & 78 & $1.2 \times 10^{-6}$ & $2.1 \times 10^{-6}$ & $1.0 \times 10^{-6}$ \\
\hline
\end{tabular}




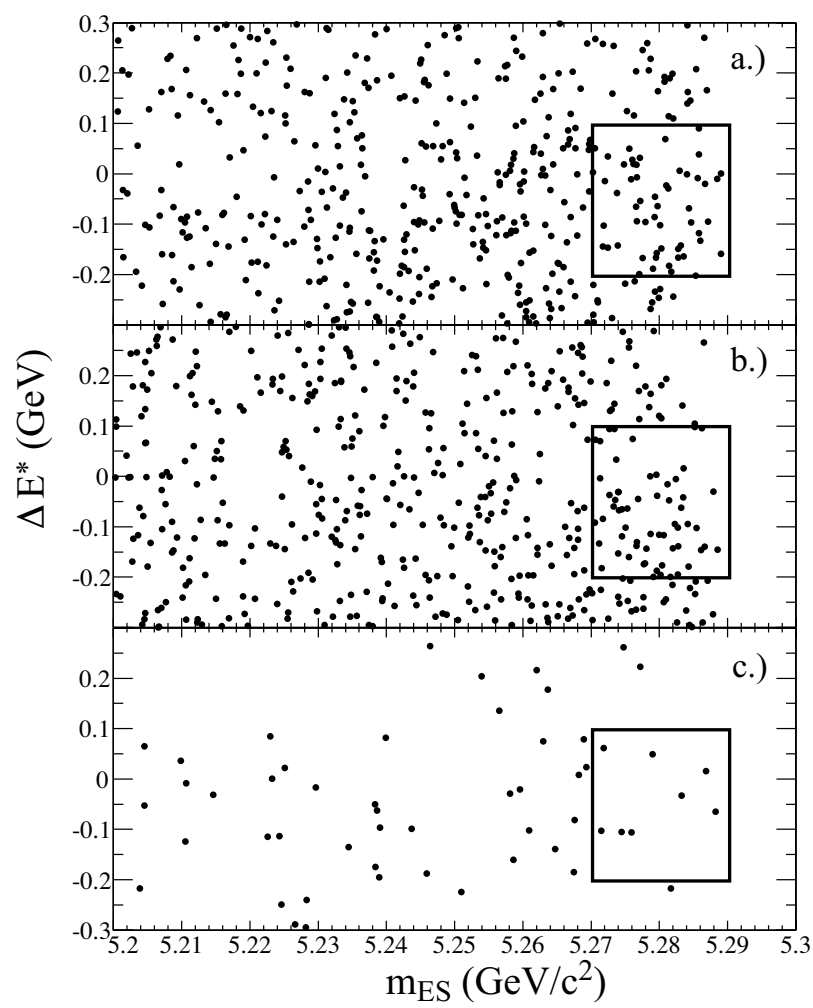

Figure 3: $\triangle E^{*}$ vs. $m_{E S}$ for BABAR data: (a) $B^{0} \rightarrow \rho^{0} \gamma$, (b) $B^{+} \rightarrow \rho^{+} \gamma$, (c) $B^{0} \rightarrow \omega \gamma$ candidates after all selection cuts. For $\mathcal{B}\left(B^{+} \rightarrow \rho^{+} \gamma\right)=1.0 \times 10^{-6}$ (and the others half as large), one would expect 5.0, 6.0 and 1.7 signal events in the three signal boxes, compared to 49, 72 , and 9 background events, respectively.

Belle quotes a combined limit on $\mathcal{B}(B \rightarrow(\rho+\omega) \gamma) \equiv \mathcal{B}\left(B^{+} \rightarrow \rho^{+} \gamma\right)=2 \mathcal{B}\left(B^{0} \rightarrow \rho^{0} \gamma\right)=$ $2 \mathcal{B}\left(B^{0} \rightarrow \omega \gamma\right)$ of $3.0 \times 10^{-6}(90 \% \mathrm{CL})$. Their 5 -mode analysis (including two $K^{*} \gamma$ charge states) then implies $\mathcal{B}(B \rightarrow(\rho+\omega) \gamma) / \mathcal{B}\left(B \rightarrow K^{*} \gamma\right)<0.081$ (90\% CL).

$B A B A R$ combines just the two $\rho$ modes, finding $\mathcal{B}(B \rightarrow \rho \gamma) \equiv \mathcal{B}\left(B^{+} \rightarrow \rho^{+} \gamma\right)=2 \mathcal{B}\left(B^{0} \rightarrow \rho^{0} \gamma\right)<$ $1.9 \times 10^{-6}(90 \% \mathrm{CL})$, which is just above the high end of the range of Standard Model predictions. With the published $B A B A R$ value for $B \rightarrow K^{*} \gamma$, this implies $\mathcal{B}(B \rightarrow \rho \gamma) / \mathcal{B}\left(B \rightarrow K^{*} \gamma\right)<0.047$ (90\% CL).

\section{$2.2 \quad$ Higher $K^{*}$ Resonances}

Belle has published measurements of $K \pi \gamma$ and $K \pi \pi \gamma B$ decays [12] using $29.4 \mathrm{fb}^{-1}$ of data. After continuum suppression (topological) cuts and a $\Delta E^{*}$ cut, the $K \pi \gamma$ signal peak in $M_{b c}$ was fitted to a sum of $K_{2}^{*}(1430), K^{*}(1410)$ and a non-resonant contribution, using $M_{K \pi}$ and the decay helicity angle $\cos \theta_{H}^{*}$ to distinguish them. Figure 4 shows the results.

Only the $K_{2}^{*}(1430)$ component was significant, with the fit implying $\mathcal{B}\left(B^{0} \rightarrow K_{2}^{*}(1430)^{0} \gamma\right)=$ $(1.3 \pm 0.5 \pm 0.1) \times 10^{-5}$. Predictions cover a wide range; this result is consistent with those from a relativistic form factor model 13 .

Belle also found $\mathcal{B}\left(B^{+} \rightarrow K^{+} \pi^{-} \pi^{+} \gamma\right)=\left(2.4 \pm 0.5_{-0.2}^{+0.4}\right) \times 10^{-5}$, dominated by $K^{* 0} \pi^{+}$and $K^{+} \rho^{0}$. 

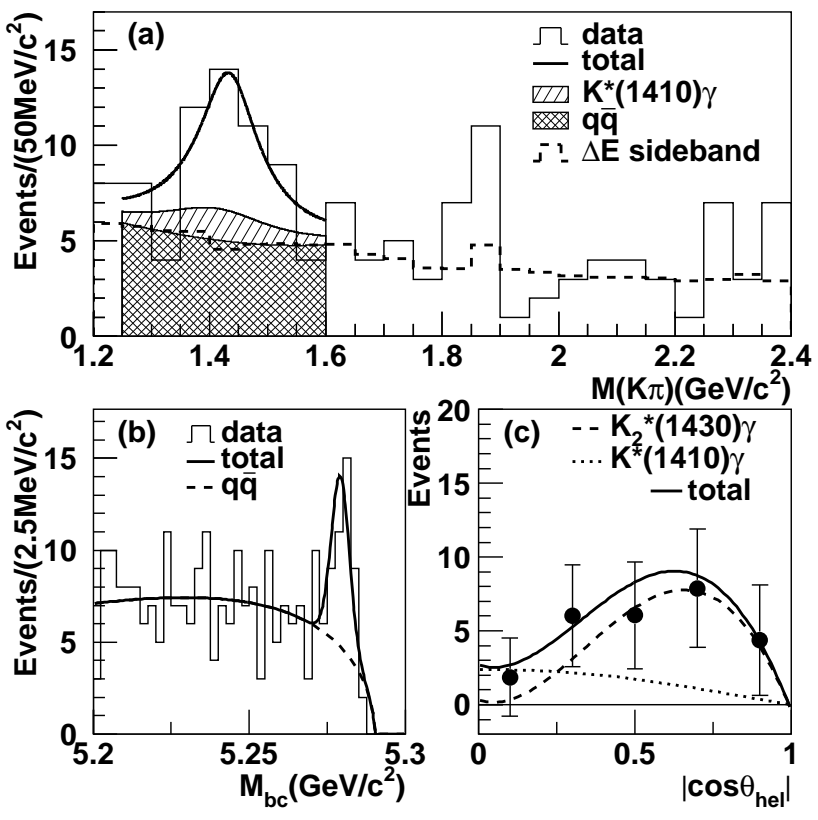

Figure 4: Distribution of Belle $B \rightarrow K^{+} \pi^{-} \gamma$ candidates: (a) $M_{K \pi}$ (for $M_{b c}>5.27 \mathrm{GeV} / c^{2}$ ), (b) $M_{b c}$ (for $1.25<M_{K \pi}<1.6 \mathrm{GeV} / c^{2}$ ) and (c) $\cos \theta_{H}^{*}$ (signal only, with $M_{b c}>5.27 \mathrm{GeV} / c^{2}$ ). The curves in (a) and (c) are results of an unbinned maximum likelihood fit.

\section{$2.3 \quad$ Inclusive $B \rightarrow X_{s} \gamma$}

Inclusive $B \rightarrow X_{s} \gamma$ measurements are of interest for several reasons:

- The NLO Standard Model computation of the branching fraction [1, 2] has precision below $10 \%$, much better than for exclusive channels. A measurement of $\mathcal{B}\left(B \rightarrow X_{s} \gamma\right)$ is sensitive to new physics.

- The $E_{\gamma}$ spectrum has been parameterized [14] in terms of just the $b$ quark mass $\left(m_{b}\right)$ and a Fermi momentum parameter $\left(\lambda_{1}\right)$. This spectrum is largely insensitive to new physics.

- The spectrum and especially its moments are related to those in $B \rightarrow X \ell \nu$, and hence relevant to the extraction of $\left|V_{c b}\right|$ and $\left|V_{u b}\right|$ in semileptonic $B$ decay.

Two independent preliminary BABAR measurements of $B \rightarrow X_{s} \gamma$, were presented at ICHEP2002 - one fully inclusive [15, the other using a semi-inclusive sum of exclusive modes [16. Because there have been no more recent results, I just report the overall situation as of ICHEP-2002.

Figure [5] summarizes the branching fraction measurements, most with a model-dependence uncertainty, denoted " \pm theo". Experiments differ in the minimum value of $E_{\gamma}$ used for analysis (a consequence of differences in background severity). Thus extrapolations to lower $E_{\gamma}$ (higher $m_{X_{s}}$ ), using the Kagan-Neubert (KN) model [14, have differing model-dependence uncertainty, largely from the choice of $m_{b}$. The BABAR semi-inclusive measurement fits the measured hadronic mass spectrum rather than extrapolating, but its model-dependence error is likewise due to limited knowledge of the KN parameters. [Two caveats at the less-than-3\% level: first, there is a lack 
of standardization of the point extrapolated to; second, the KN model may have omitted a small radiative effect [17] in the extrapolation.] Results to date are in agreement with the Standard Model prediction.

The mean $\left\langle E_{\gamma}>\right.$ above the measured $E_{\gamma}$ cutoff can be related to $\bar{\Lambda}$ in HQET, using a procedure from Ligeti et al. [18. Figure [6 summarizes values of $\bar{\Lambda}$ obtained from either $B \rightarrow X_{s} \gamma$ or semi-leptonic decays. Uncertainties are still large.

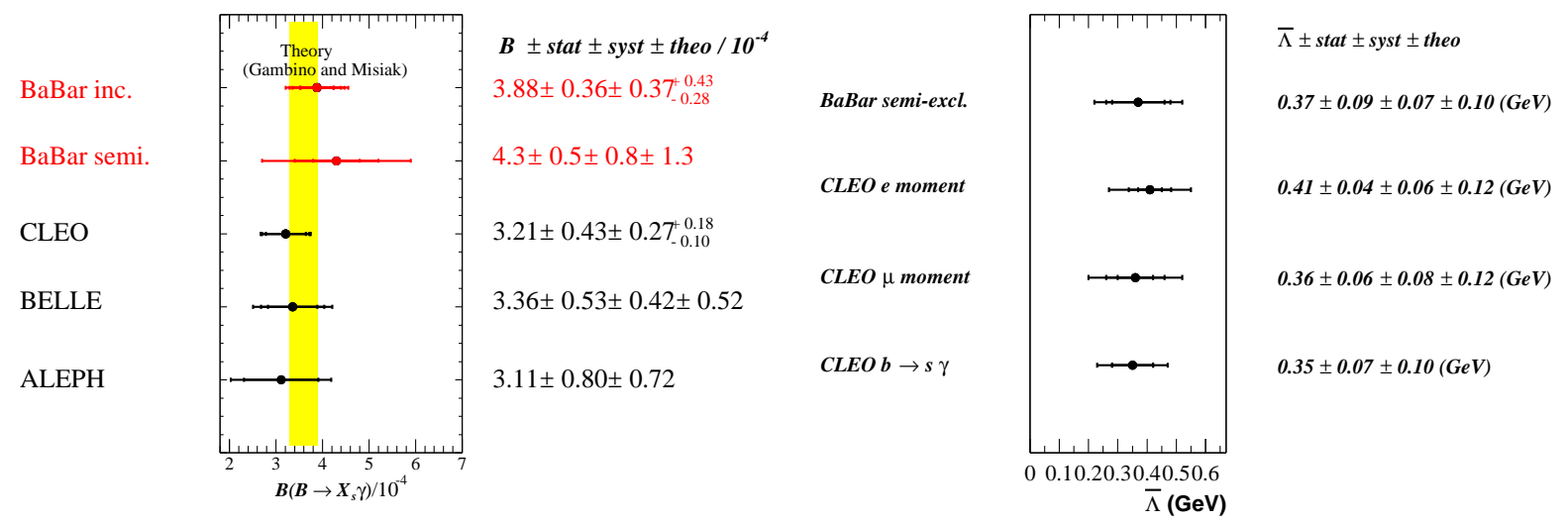

Figure 5: Measurements of $\mathcal{B}\left(B \rightarrow X_{s} \gamma\right)$ compared to the NLO Standard Model prediction (shaded band). See "theo" explanation and caveats in text.

Figure 6: Measurements of $\bar{\Lambda}$ in HQET (in $\mathrm{GeV}$ ) from $B \rightarrow X_{s} \gamma$ and semi-leptonic $B$ decays.

\section{Decays Resulting in Charged-Lepton Pairs}

Detecting a lepton pair $\left(e^{+} e^{-}\right.$or $\left.\mu^{+} \mu^{-}\right)$instead of a photon opens up a new dimension (the lepton pair mass), and adds the box and " $Z$ penguin" amplitudes (Fig. 2), providing more ways in which new physics might enter. $B \rightarrow X_{s} \gamma$ is sensitive mainly to the magnitude of the Wilson coefficient $C_{7}$ in the Operator Product Expansion, while $B \rightarrow X_{s} \ell^{+} \ell^{-}$is sensitive also to its phase and to coefficients $C_{9}$ and $C_{10}$. The penalty one pays is an expected branching fraction 50 or so times smaller in the Standard Model. As with $B \rightarrow X_{s} \gamma$, experimenters began with exclusive modes, but have already moved on to the inclusive measurement.

\section{1 $\quad B \rightarrow K^{(*)} \ell^{+} \ell^{-}$}

Belle [19] first established a signal for $K \ell^{+} \ell^{-}$from $29 \mathrm{fb}^{-1}$. The most recent Belle [20] and BABAR 21] results were presented at ICHEP in 2002. Eight modes are measured: $K / K^{*}$, charged/neutral, $e^{+} e^{-} / \mu^{+} \mu^{-}$. Both experiments veto backgrounds consistent with $J / \psi\left(\psi^{\prime}\right) \rightarrow \ell^{+} \ell^{-}$(allowing also for Bremsstrahlung in the case of $e^{+} e^{-}$) and suppress continuum and combinatoric $B \bar{B}$ backgrounds with topological and kinematic cuts. They then fit to separate the signal from the residual background. Spectra and fits are shown in Figures 7 and 8.

The $K^{+}$and $K^{0}$ modes are combined by assuming isospin symmetry. Lepton universality is assumed for $K \ell^{+} \ell^{-}$. But $K^{*} \ell^{+} \ell^{-}$has a photon pole at $m_{\ell^{+} \ell^{-}}^{2}=0$, resulting in $\mathcal{B}(B \rightarrow$ $\left.K^{*} e^{+} e^{-}\right) / \mathcal{B}\left(B \rightarrow K^{*} \mu^{+} \mu^{-}\right)>1$. Belle constrains this ratio to 1.33, from Ali et al. [22]; while 

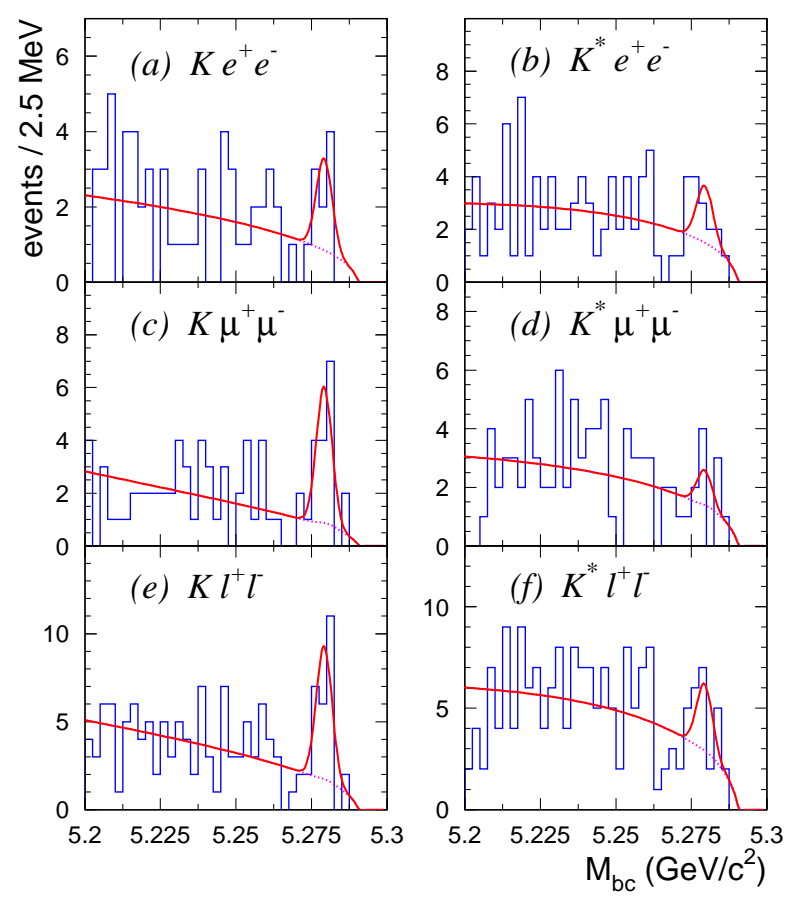

Figure 7: Belle preliminary spectra (ICHEP$\left.2002,60.1 \mathrm{fb}^{-1}\right)$ for $B \rightarrow K^{(*)} \ell^{+} \ell^{-}$after $\Delta E^{*}$ cuts, summed over charge states. The spectra in (e) and (f) are the sums of those above. The curves show binned maximum likelihood fits to each $M_{b c}$ spectrum.
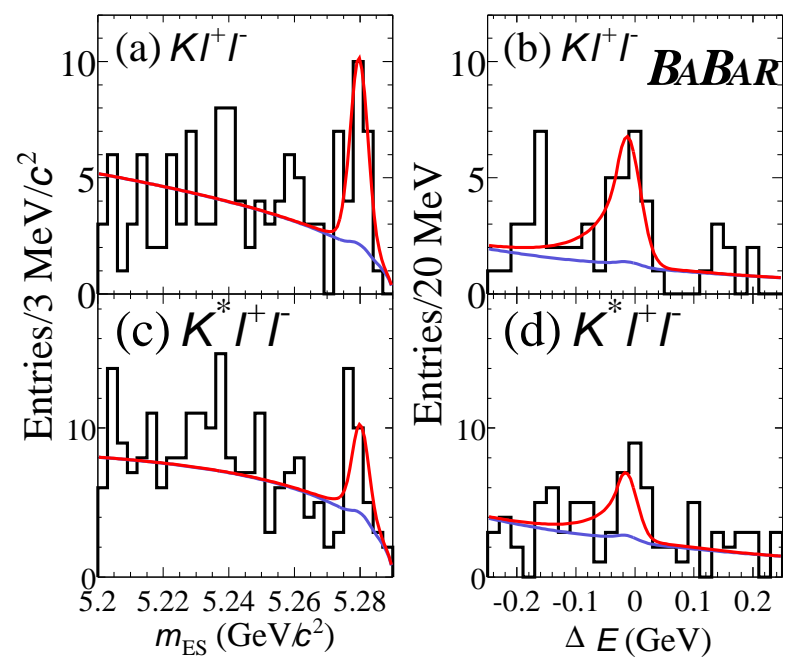

Figure 8: BABAR preliminary spectra (ICHEP$2002,77.8 \mathrm{fb}^{-1}$ ) for $B \rightarrow K^{(*)} \ell^{+} \ell^{-}$summed over charge states and lepton flavor. The curves are projections of unbinned two-dimensional maximum likelihood fits to $m_{E S}$ and $\Delta E^{*}$, with relative branching fractions for the four component processes constrained (see text).

BABAR used a ratio of 1.21 from an earlier paper 23. Results are shown in Table 2. The NNLO Standard Model prediction from Ali et al. [22] is $\mathcal{B}\left(B \rightarrow K \ell^{+} \ell^{-}\right)=(0.35 \pm 0.13) \times 10^{-6}$, more than $1 \sigma$ below each measurement, but there are higher predictions in the literature, and no significant disagreement can be claimed.

\subsection{A First Measurement of $B \rightarrow X_{s} \ell^{+} \ell^{-}$}

As with $B \rightarrow X_{s} \gamma, B \rightarrow X_{s} \ell^{+} \ell^{-}$can be much more precisely computed in the Standard Model than can its exclusive counterparts. Belle has now published a first measurement 24 for dilepton masses above $0.2 \mathrm{GeV} / c^{2}$. The NNLO prediction of Ali et al. 22] is $\mathcal{B}\left(B \rightarrow X_{s} \ell^{+} \ell^{-}\right.$with $M_{\ell^{+} \ell^{-}}>$ $\left.0.2 \mathrm{GeV} / c^{2}\right)=(4.2 \pm 0.7) \times 10^{-6}$.

Belle's measurement is semi-inclusive, using 18 specific final states (one $K^{+}$or $K_{S}^{0}, 0$ to 4 $\pi$, including 0 or $1 \pi^{0}$ ). In order to estimate signal detection efficiencies and optimize selection cuts, the Monte Carlo detector simulation uses theoretical models as input for the dilepton mass spectrum 22 and the hadronic mass $\left(M_{X_{s}}\right)$ Fermi spectrum [25, while final hadronic states are produced according to JETSET. For $M_{X_{s}}<1.1 \mathrm{GeV} / c^{2}$, the exclusive-state predictions [22] are used.

Topological and $\Delta E^{*}$ (Eq. 1) cuts are used to reduce substantial backgrounds from continuum events and from random combinations of leptons from semileptonic $B$ decays; $M_{X_{s}}<2.1 \mathrm{GeV} / c^{2}$ 
Table 2: Belle and BABAR preliminary (ICHEP-2002) results for $B \rightarrow K^{(*)} \ell^{+} \ell^{-}$. Note: the Belle limit for $K^{*} \ell^{+} \ell^{-}$is an average of $e^{+} e^{-}$and $\mu^{+} \mu^{-}$modes, while the BABAR limit is scaled to $e^{+} e^{-}$. If the $B A B A R K^{*} \ell^{+} \ell^{-}$result were interpreted as a signal, its value would be $\left(1.68_{-0.58}^{+0.68} \pm 0.28\right) \times 10^{-6}$, but its significance is only $2.8 \sigma$.

\begin{tabular}{|l|c|c|}
\hline Branching Ratios & Belle $\times 10^{6}$ & $B A B A R \times 10^{6}$ \\
\hline$B \rightarrow K \mu^{+} \mu^{-}$ & $0.80_{-0.23}^{+0.28} \pm 0.09$ & \\
$B \rightarrow K \ell^{+} \ell^{-}$ & $0.58_{-0.15}^{+0.17} \pm 0.06$ & $0.78_{-0.20}^{+0.24}{ }_{-0.18}^{+0.11}$ \\
\hline$B \rightarrow K^{*} \ell^{+} \ell^{-}$ & $<1.4(90 \% \mathrm{CL})$ & $<3.0(90 \% \mathrm{CL})$ \\
\hline
\end{tabular}

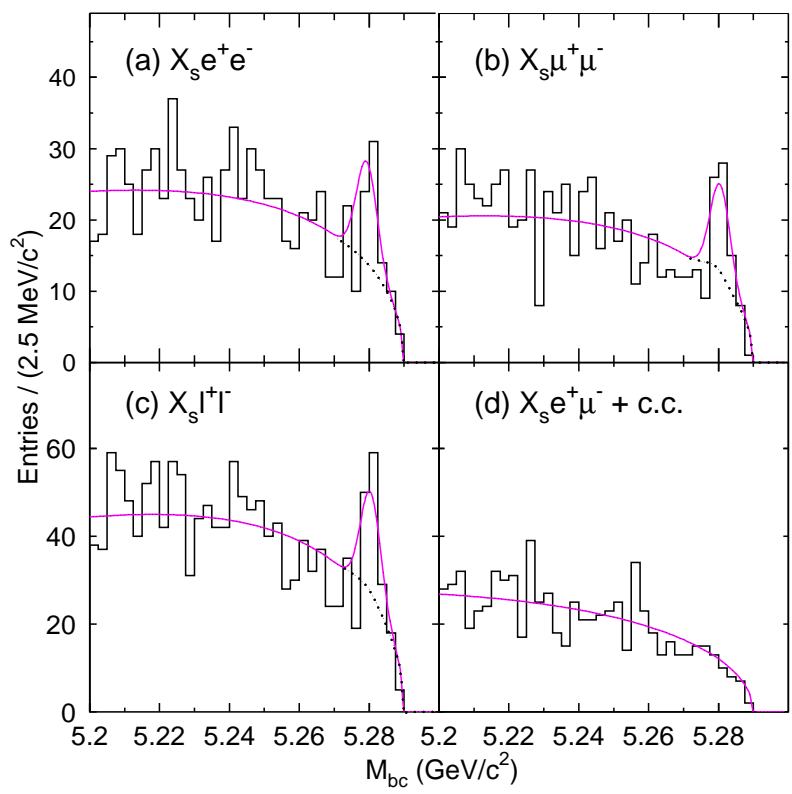

Figure 9: Beam-constrained mass distributions for Belle measurement of $B \rightarrow X_{s} \ell^{+} \ell^{-}$ $\left(60 \mathrm{fb}^{-1}\right)$ : (a) $e^{+} e^{-}$, (b) $\mu^{+} \mu^{-}$, (c) sum of $e^{+} e^{-}$ and $\mu^{+} \mu^{-}$, (d) $e^{ \pm} \mu^{\mp}$. The solid lines are the results of unbinned maximum likelihood fits, while the dashed lines show the background contributions in these fits.

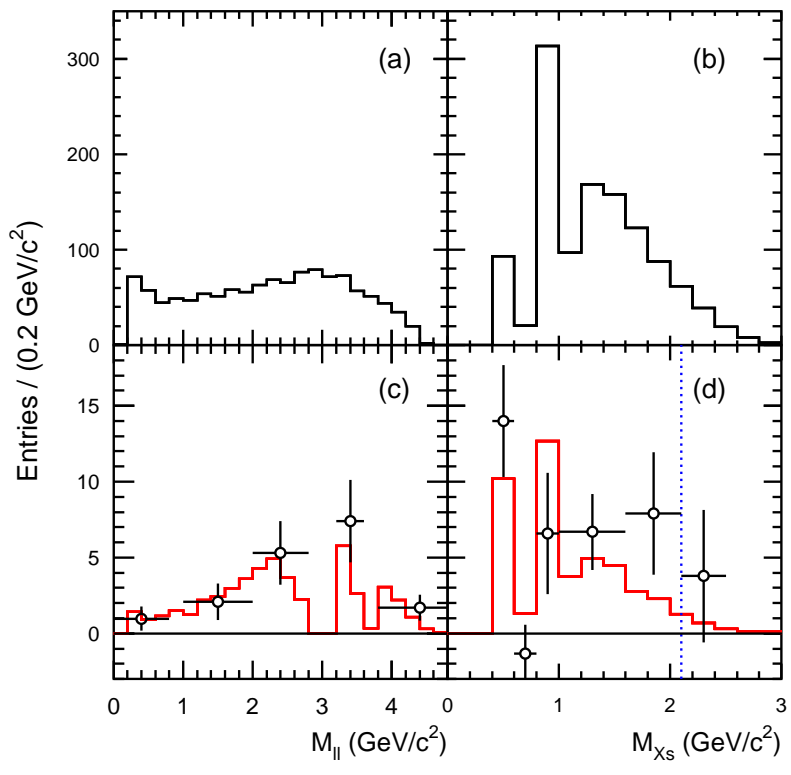

Figure 10: Standard Model expectations for the dilepton (left) and hadronic (right) mass distributions of signal events, before (top) and after (bottom) all selection cuts. Histograms are normalized to the predicted branching fraction. The data points show Belle results, with statistical errors. 
avoids a high-background region. Charmonium backgrounds (from $B \rightarrow J / \psi\left(\psi^{\prime}\right) X_{s}$ with leptonic decay of the $J / \psi$ or $\psi^{\prime}$ ) are vetoed using the dilepton mass. After cuts, the $M_{b c}$ (Eq. 2) spectra are fitted to a Gaussian signal plus an ARGUS background shape 9], as shown in Figure 9. A signal is observed in the $e^{+} e^{-}$and $\mu^{+} \mu^{-}$channels, but is absent (as expected) for $e^{ \pm} \mu^{\mp}$. Finally, a small background from $X_{s} \pi^{+} \pi^{-}$with double pion misidentification (2.6 events for $\mu^{+} \mu^{-}$) is subtracted from the fit signal.

Combining the $e^{+} e^{-}$and $\mu^{+} \mu^{-}$results, Belle finds 57.4 net signal events, with a significance of $5.4 \sigma$. Averaging the two modes, they find $\mathcal{B}\left(B \rightarrow X_{s} \ell^{+} \ell^{-}\right)=\left[6.1 \pm 1.4\right.$ (stat) ${ }_{-1.1}^{+1.4}($ syst $\left.)\right] \times 10^{-6}$, where the systematic uncertainty includes $\approx 0.8$ from model-dependence in $K$ and $K^{*}$ fractions, the $m_{X_{s}}$ model, and quark fragmentation. This result is in agreement with the Standard Model expectations. The distributions of events in dilepton and hadronic masses, shown in Figure 10, are also compatible with expectations.

\section{A Search for $\mathbf{B}^{-} \rightarrow \mathbf{K}^{-} \nu \bar{\nu}$}

The process $B^{-} \rightarrow K^{-} \nu \bar{\nu}$ occurs in the SM via the same lowest-order diagrams as $B \rightarrow X_{s} \ell^{+} \ell^{-}$ (Fig. 2), but only $Z^{0}$ and $W^{ \pm}$, not $\gamma$, can contribute, i.e., there are no long-range effects. As a consequence, the SM computation is relatively clean for the corresponding inclusive process. As usual, SM predictions [26, 27] for the exclusive process $B^{-} \rightarrow K^{-} \nu \bar{\nu}$ have additional hadronization uncertainties. Summing over three neutrino flavors, Buchalla et al. [26] predict $\mathcal{B}\left(B^{-} \rightarrow K^{-} \nu \bar{\nu}\right)=$ $\left(3.8_{-0.6}^{+1.2}\right) \times 10^{-6}$. Models with altered Flavor-Changing-Neutral-Current $Z^{0}$ couplings (e.g., SUSY or dynamical Higgs) can increase this by up to a factor of five. However, the measurement of $B \rightarrow X_{s} \ell^{+} \ell^{-}$(section [3.2) already provides tighter constraints.

The lowest published limit, from CLEO [28], is $2.4 \times 10^{-4}$. Last year BABAR [29] set a preliminary limit of $9.4 \times 10^{-5}$, based on $50.7 \mathrm{fb}^{-1}$ of data and using semi-leptonic tagging of the other $B$.

The new BABAR analysis [30] to be described here, based on $80.1 \mathrm{fb}^{-1}$, is entirely independent statistically. It relies on an orthogonal tagging sample, using full reconstruction of the other $B$ via $B^{+} \rightarrow \bar{D}^{0} X^{+}$, where $X^{+}$is composed of up to three charged mesons ( $\pi$ or $K$ ) and up to two $\pi^{0}$ s. The $\bar{D}^{0}$ is reconstructed in $K^{+} \pi^{-}, K^{+} \pi^{-} \pi^{0}$ or $K^{+} \pi^{-} \pi^{+} \pi^{-}$.

Continuum backgrounds are reduced by topological cuts. $\Delta E^{*}$ and $m_{E S}$ for the tag candidates (see Eqs. 1]and 2), are required to be between -1.8 and $0.6 \mathrm{GeV}$, and above $5.2 \mathrm{GeV} / c^{2}$, respectively. Any ambiguity is resolved by choosing the candidate with $\Delta E^{*}$ closest to 0 . The resulting simulated $m_{E S}$ spectra show a clear peak only for $B^{+} B^{-}$events. Events with $5.272<m_{E S}<5.288 \mathrm{GeV} / c^{2}$ are then used to search for the signal, using everything not included in the reconstructed tag. Signal-side requirements are: only one charged track (opposite charge to the tag), identified as a kaon; kaon momentum at least $1.5 \mathrm{GeV} / c$ in the $\mathrm{CM}$; the total missing momentum vector pointing into the detector acceptance; no pair of calorimeter clusters consistent with a $\pi^{0}$ mass; and total extra neutral CM-frame energy $E_{\text {extra }}<300 \mathrm{MeV}$. The latter is a particularly powerful cut, as can be seen in Figure 11], The overall efficiency for signal events is $(0.0458 \pm 0.0046) \%$.

A combinatoric background of $1.0 \pm 0.4$ events is estimated by extrapolating from an $m_{E S}$ sideband, while a peaking background of $1.7 \pm 0.6$ events is taken from $B^{+} B^{-}$Monte Carlo simulation. The total background of $2.7 \pm 0.7$ compares to 3 data events in the unblinded signal region.

By using a large number of parameterized ("toy") Monte Carlo experiments for each signal hypothesis, a preliminary upper limit of $\mathcal{B}\left(B^{-} \rightarrow K^{-} \nu \nu\right)<1.05 \times 10^{-4}$ (90\% CL) is found. The efficiency quoted above is based on the nominal $K$ momentum spectrum given by Buchalla et al. [26] Using their extreme spectra scales the upper limit to 1.10 or 1.02 , while using the curve in 

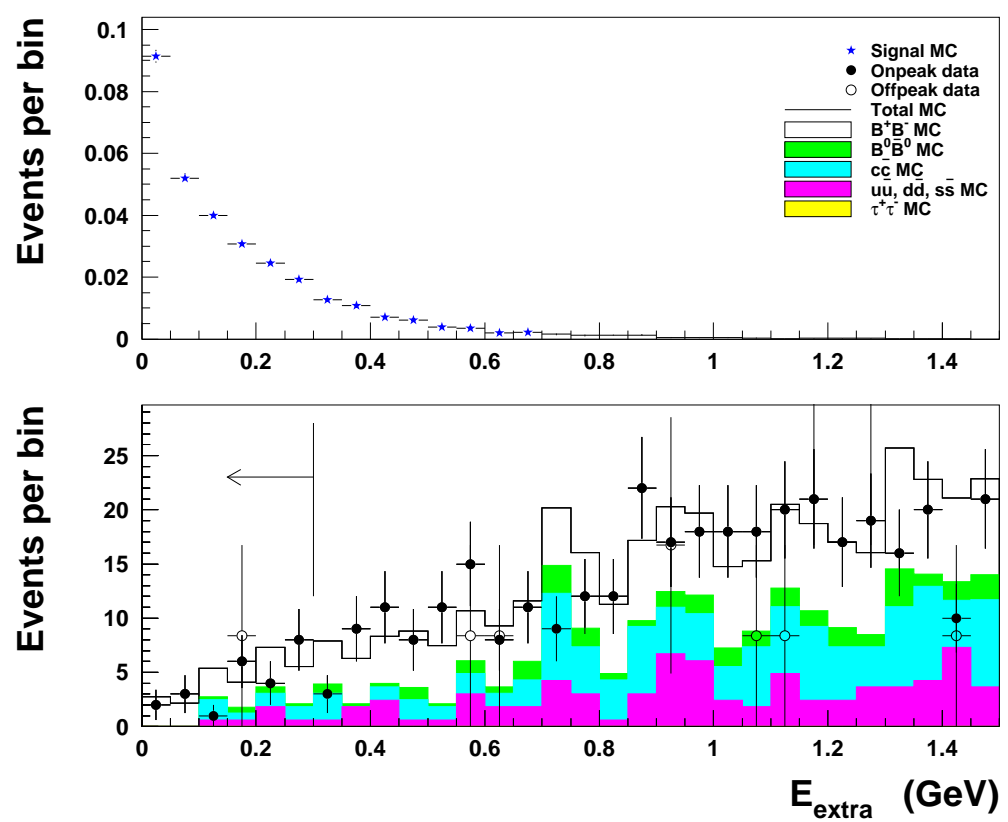

Figure 11: Simulated (Monte Carlo) distributions of "extra" neutral energy (see text) for the BABAR $B^{-} \rightarrow K^{-} \nu \bar{\nu}$ search. The top plot is for signal, assuming $\mathcal{B}\left(B^{-} \rightarrow K^{-} \nu \bar{\nu}\right)=4.0 \times 10^{-6}$, while the bottom plot shows cumulative continuum and generic $B \bar{B}$ backgrounds. Both are scaled to the luminosity of the data, and are after the signal-side requirements of an identified kaon and no other tracks, but no other signal-side cuts.

Faessler et al. 27] results in $0.95\left(\right.$ all $\left.\times 10^{-4}\right)$. Combining the nominal result with the earlier, independent, BABAR measurement [29] results in $\mathcal{B}\left(B^{-} \rightarrow K^{-} \nu \nu\right)<7.0 \times 10^{-5}$.

\section{$5 \quad$ Searches for $\mathbf{B}^{+} \rightarrow \tau^{+} \nu_{\tau}$}

Purely leptonic $B^{+}$decays in the SM occur via an s-channel $W^{+}$and have cleanly computed branching fractions:

$$
\mathcal{B}\left(B^{+} \rightarrow \ell^{+} \nu\right)=\frac{G_{F}^{2} m_{B} m_{\ell}^{2}}{8 \pi}\left(1-\frac{m_{\ell}^{2}}{m_{B}^{2}}\right)^{2} f_{B}^{2}\left|V_{u b}\right|^{2} \tau_{B}
$$

From current PDG values [3], using lattice gauge theory for $f_{B}, \mathcal{B}\left(B^{+} \rightarrow \tau^{+} \nu_{\tau}\right) \approx 7.5 \times 10^{-5}$. (The $\mu^{+} \nu_{\mu}$ final state would be much easier to measure, but its prediction is $\approx 250 \times$ smaller, a result of "helicity suppression", i.e., the $\ell^{+}$is produced left-handed.) Depending on the state of external knowledge of $f_{B}\left|V_{u b}\right|$, a measurement could either provide this product (within the SM) or provide evidence for new physics (e.g., a charged Higgs, or leptoquark exchange). The most stringent published limit is from L3 [31], $<5.7 \times 10^{-4}$ at $90 \%$ CL.

$B A B A R$ now has two new independent preliminary results, both using $81.9 \mathrm{fb}^{-1}$ of data:

- One 32 uses semi-leptonic $B$ decays as tags, and considers only the leptonic decay modes of the $\tau^{+}$, i.e., $e^{+} \nu_{e} \bar{\nu}_{\tau}$ and $\mu^{+} \nu_{\mu} \bar{\nu}_{\tau}$. 
- The other [33] uses fully-reconstructed $B$ s for tagging, and considers five $\tau^{+}$modes, adding $\pi^{+} \bar{\nu}_{\tau}, \pi^{+} \pi^{0} \bar{\nu}_{\tau}$, and $\pi^{+} \pi^{-} \pi^{+} \bar{\nu}_{\tau}$.

Because the basics of the second method are similar to those of the BABAR $K^{-} \nu \bar{\nu}$ analysis (sec. 4), this summary focuses on the quite different semi-leptonic-tag method. The $\tau^{+}$is observed via its single charged lepton; nothing is missing except neutrinos. Hence any other tracks or neutral energy must arise from the other (tagging) $B^{-}$. Keeping only events with a total event charge of 0 , this "tag" is reconstructed as $B^{-} \rightarrow D^{0} \ell^{-} \bar{\nu}_{\ell} X$, beginning with a reconstructed $D^{0}$ in a $K^{-} \pi^{+}$, $K^{-} \pi^{+} \pi^{0}, K^{-} \pi^{+} \pi^{+} \pi^{-}$or $K_{S}^{0} \pi^{+} \pi^{-}$mode, and vertexing this with a high-momentum lepton. If there are multiple $D \ell$ candidates, the best is selected using the $D^{0}$ mass. If $X$ is assumed to be null, there is enough information to compute the cosine of the angle between the parent $B$ and the $D \ell$. If the assumption is wrong, this cosine can take on values beyond \pm 1 ; a generous cut allows for feeddown from higher-mass neutral $D$ states (e.g., from $D^{* 0} \rightarrow D^{0} \pi^{0}$ ) while still suppressing background.

Anything remaining after tag reconstruction is assigned to the "signal side". There must be a single charged track, identified as $e^{+}$or $\mu^{+}$. Additional cuts reject $\tau^{+} \tau^{-}$continuum events. The total "signal-side" neutral energy $E_{l e f t}$ is the signal-defining quantity in this analysis. (For a real $\tau^{+} \nu_{\tau}$ event, non-zero $E_{\text {left }}$ can arise from beam backgrounds or from unused particles contributing to $X$ in the decay of the tag $B$.) Figure 12 shows distributions for signal and background MC simulation, along with data in the background region. An extended maximum likelihood fit is then used to fit the data to signal plus background between 0 and $1 \mathrm{GeV}$; the background simulation predicts 269 events in this fit region for the BABAR data sample.

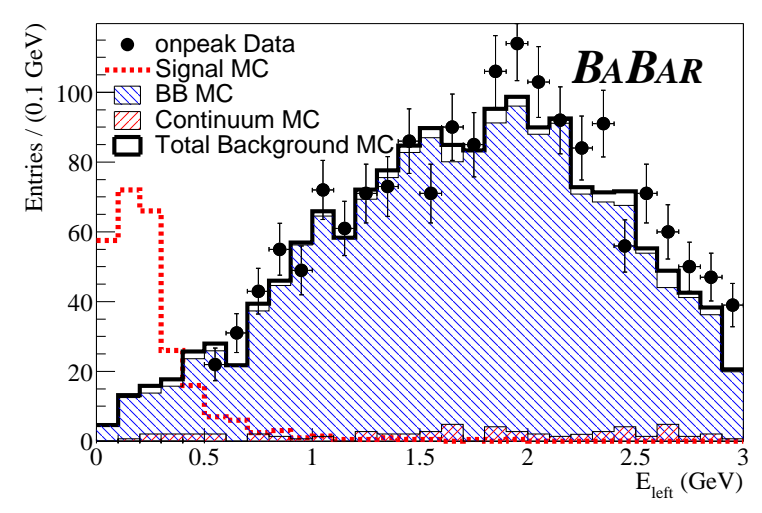

Figure 12: $E_{\text {left }}$ after all cuts for BABAR measurement of $B^{+} \rightarrow \tau^{+} \nu_{\tau}$ using semi-leptonic tags (preliminary). The signal MC has arbitrary normalization. (Data points below $0.5 \mathrm{MeV}$ were kept hidden at this stage, until systematic studies and fitting and and limitsetting procedures were settled.)

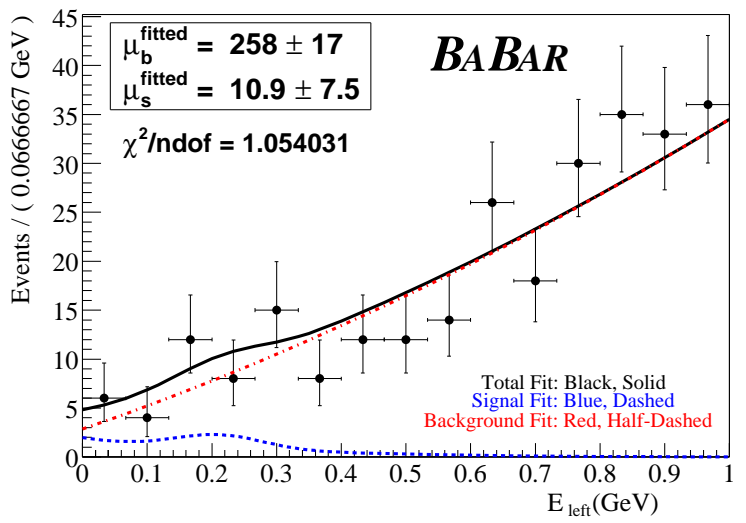

Figure 13: $E_{\text {left }}$ distribution of data (no longer hidden) after all cuts for $B A B A R$ measurement of $B^{+} \rightarrow \tau^{+} \nu_{\tau}$ using semi-leptonic tags (preliminary). Curves show the maximum likelihood fit, along with its signal and background components. The quoted $\mu$ values are the fitted numbers of events.

The fitted signal is converted to a 90\% CL upper limit using a version of the approach 34] devised for Higgs searches at LEP, in this case implemented via many "toy" MC experiments for each signal hypothesis. The median upper limit for background-only toy-MC is 15.4 events, which can be regarded as the sensistivity of this measurement. Two independent dataset-sized backroundonly full simulations were run in order to study systematic effects. The "Higgs procedure" applied 
to these yielded upper limits of 13.3 and 19.4 events, close to the sensitivity. These full simulations were also used to study possible variations in the signal and background $E_{\text {left }}$ distribution functions, with the conclusion that the default analysis is conservative for the purpose of setting an upper limit.

Figure 13 shows the results of the $E_{\text {left }}$ fit. The 10.9 fitted signal events translate to a 90\% CL limit of 22.8 (well within the spread of background-only toy-MC outputs). Taking into account the signal efficiency and its systematic uncertainty (determined using data control samples), the result is $\mathcal{B}\left(B^{+} \rightarrow \tau^{+} \nu_{\tau}\right)<4.9 \times 10^{-4}(90 \% \mathrm{CL}$, preliminary). The independent BABAR measurement using fully-reconstructed tags resulted in $\mathcal{B}\left(B^{+} \rightarrow \tau^{+} \nu_{\tau}\right)<7.7 \times 10^{-4}$ (90\% CL, preliminary). The combined BABAR upper limit is then $\mathcal{B}\left(B^{+} \rightarrow \tau^{+} \nu_{\tau}\right)<4.1 \times 10^{-4}$ (90\% CL, preliminary). This is still a factor of 5.5 above the SM prediction, but is somewhat lower than the previous best limit.

\section{Conclusions}

Rare $B$ decays have the potential for exhibiting physics beyond the SM, but no deviation has yet been demonstrated. Limits on several unseen exclusive decay modes $\left(\rho \gamma, \omega \gamma, K^{-} \nu \bar{\nu}, \tau^{+} \nu_{\tau}\right)$ have come down significantly since last year's Moriond EW conference, and it is important to push them (and others, like $\mu^{+} \nu_{\mu}$ ) further, since non-SM effects could in some cases enter at levels significantly above the SM predictions. The limits for $B \rightarrow \rho(\omega) \gamma$ (section 2.1) are now only a factor of two above the low end of SM predictions, so we may be close to observing a $b \rightarrow d \gamma$ signal for the first time.

Meanwhile, the first observation of inclusive $B \rightarrow X_{s} \ell^{+} \ell^{-}$(section [3.2) opens up a rich new area of investigation. The exclusive channels $K^{(*)} \ell^{+} \ell^{-}$(section [3.1, with the $K$ mode established, the $K^{*}$ mode nearly so) are already offering some tantalizing SM comparisons, albeit with the usual caveat that predictions for exclusive modes are considerably more uncertain than those for inclusive modes. Measurements of inclusive $X_{s} \gamma$ (section 2.3) are moving toward useful precision on the $E_{\gamma}$ spectrum and its moments, which are linked theoretically to semileptonic decays; while the branching fraction values already show that any SM deviation in this sector (more limited than that accessible with $X_{s} \ell^{+} \ell^{-}$) must be fairly small. Finally, the first published measurement on radiative decay to a higher $K^{*}$ resonance (section 2.2) marks the start of an exploration of the detailed composition of the inclusive $B \rightarrow X_{s} \gamma$ process

Both BABAR and Belle are continually updating results to new data and improving analysis techniques, and we can expect continued progress in all of the above areas and more.

\section{Acknowledgments}

The author thanks the physicists of the Belle collaboration and the BABAR collaboration (of which he is a member) for providing the figures and detailed explanations needed to prepare this report. Its preparation was supported in part by the U.S. Department of Energy, \#DE-FG03-92ER40689.

\section{References}

[1] P. Gambino and M Misiak, Nucl. Phys. B 611, 338 (2001).

[2] A.J. Buras and M. Misiak, Acta Phys. Polon. B 33, 2597 (2002). 
[3] Particle Data Group, Phys. Rev. D 66, 010001 (2002).

[4] A. Ali and A.Y. Parkhomenko, Eur. Phys. J. C 23, 89 (2002).

[5] S.W. Bosch and G. Buchalla, Nucl. Phys. B 621, 459 (2002).

[6] B. Aubert et al., BABAR Collaboration, SLAC-PUB-9938, BABAR-PUB-03/008 (2003).

[7] K. Abe et al., Belle Collaboration, BELLE-CONF-0240 (2002); results updated via private communication, T. Browder and M. Nakao.

[8] B. Aubert et al., BABAR Collaboration, Nucl. Instrum. Methods A 479, 1 (2002).

[9] H. Albrecht et al., ARGUS Collaboration, Z. Phys. C 48, 543 (1990).

[10] R. Cousins and V. Highland, Nucl. Instrum. Methods A 320, 331 (1992).

[11] T.E. Coan et al., CLEO Collaboration, Phys. Rev. Lett. 84, 5283 (2000).

[12] S. Nishida et al., Belle Collaboration, Phys. Rev. Lett. 89, 231801 (2002).

[13] S. Veseli and M.G. Olsson, Phys. Lett. B 367, 309 (1996).

[14] A.L. Kagan and M. Neubert, Eur. Phys. J. C 7, 5 (1999).

[15] B. Aubert et al., BABAR Collaboration, SLAC-PUB-9301, BABAR-CONF-02/26 (2002).

[16] B. Aubert et al., BABAR Collaboration, SLAC-PUB-9308, BABAR-CONF-02/25 (2002).

[17] I. Bigi and N. Uraltsev, Int. J. Mod. Phys. A 17, 4709 (2002).

[18] Z. Ligeti, M.E. Luke, A.V. Manohar and M.B. Wise, Phys. Rev. D 60, 034019 (1999), plus private communication from Z. Ligeti and M.B. Wise.

[19] K. Abe et al., Belle Collaboration, Phys. Rev. Lett. 88, 021802 (2002).

[20] K. Abe et al., Belle Collaboration, BELLE-CONF-0241 (2002).

[21] B. Aubert et al., BABAR Collaboration, SLAC-PUB-9323, BABAR-CONF-02/023 (2002).

[22] A. Ali, E. Lunghi, C. Greub and G. Hiller, Phys. Rev. D 66, 034002 (2002).

[23] A. Ali, P. Ball, L.T. Handoko and G. Hiller, Phys. Rev. D 61, 074024 (2000).

[24] J. Kaneko et al., Belle Collaboration, Phys. Rev. Lett. 90, 021801 (2003).

[25] A. Ali, G. Hiller, L.T. Handoko and T. Morozumi, Phys. Rev. D 55, 4105 (1997).

[26] G. Buchalla, G. Hiller and G. Isidori, Phys. Rev. D 63, 014015 (2001).

[27] A. Faessler et al., Eur. Phys. J. C 4, 18 (2002).

[28] T.E. Browder et al., CLEO Collaboration, Phys. Rev. Lett. 86, 2950 (2001).

[29] B. Aubert et al., BABAR Collaboration, SLAC-PUB-9309, BABAR-CONF-02/027 (2002). 
[30] B. Aubert et al., BABAR Collaboration, SLAC-PUB-9710, BABAR-CONF-03/006 (2003).

[31] M. Acciari et al., L3 Collaboration, Phys. Lett. B 396, 327 (1997).

[32] B. Aubert et al., BABAR Collaboration, SLAC-PUB-9688, BABAR-CONF-03/005 (2003).

[33] B. Aubert et al., BABAR Collaboration, SLAC-PUB-9716, BABAR-CONF-03/004 (2003).

[34] A.L. Read, First Workshop on Confidence Limits, CERN-2000-005, 81 (2000). 